\title{
Resilience of Networked Infrastructure with Evolving Component Conditions: Pavement Network Application
}

\author{
Levenberg, Eyal; Miller-Hooks, Elise; Asadabadi, Ali; Faturechi, Reza
}

Published in:

Journal of Computing in Civil Engineering

Link to article, DOI:

10.1061/(ASCE)CP.1943-5487.0000629

Publication date:

2017

Document Version

Peer reviewed version

Link back to DTU Orbit

Citation (APA):

Levenberg, E., Miller-Hooks, E., Asadabadi, A., \& Faturechi, R. (2017). Resilience of Networked Infrastructure with Evolving Component Conditions: Pavement Network Application. Journal of Computing in Civil Engineering, 31(3), [4016060]. https://doi.org/10.1061/(ASCE)CP.1943-5487.0000629

\section{General rights}

Copyright and moral rights for the publications made accessible in the public portal are retained by the authors and/or other copyright owners and it is a condition of accessing publications that users recognise and abide by the legal requirements associated with these rights.

- Users may download and print one copy of any publication from the public portal for the purpose of private study or research.

- You may not further distribute the material or use it for any profit-making activity or commercial gain

- You may freely distribute the URL identifying the publication in the public portal 


\title{
Resilience of Networked Infrastructure with Evolving Component Conditions: A Pavement Network Application
}

\author{
Eyal Levenberg ${ }^{1}$, Elise Miller-Hooks ${ }^{2}$, Ali Asadabadi $^{3}$, and Reza Faturechi ${ }^{4}$
}

Abstract: This paper deals with quantifying the resilience of a network of pavements. Calculations were carried out by modeling network performance under a set of possible damage-meteorological scenarios with known probability of occurrence. Resilience evaluation was performed a priori while accounting for optimal preparedness decisions and additional response actions that can be taken under each of the scenarios. Unlike the common assumption that the pre-event condition of all system components is uniform, fixed, and pristine, component condition evolution was incorporated herein. For this purpose, the health of the individual system components immediately prior to hazard event impact, under all considered scenarios, was associated with a serviceability rating. This rating was projected to reflect both natural deterioration and any intermittent improvements due to maintenance. The scheme was demonstrated for a hypothetical case study involving Laguardia Airport. Results show that resilience can be impacted by the condition of the infrastructure elements, their natural deterioration processes, and prevailing maintenance plans. The findings imply that, in general, upper bound values are reported in ordinary resilience work, and that including evolving component conditions is of value.

Author keywords: Resilience; Pavement; Infrastructure; Serviceability; Aging, Maintenance.

\footnotetext{
${ }^{1}$ Corresponding author, Technical University of Denmark, E-mail: eylev@byg.dtu.dk

${ }^{2}$ University of Maryland, E-mail: elisemh@umd.edu

${ }^{3}$ University of Maryland, E-mail: ali.asadabadi@gmail.com

${ }^{4}$ JetBlue, E-mail: reza.faturechi@gmail.com
} 


\section{Introduction and Motivation}

Networked civil infrastructures, such as transportation, water, and energy systems, are essential to the functioning of any modern society, and therefore must be resilient. Numerous works have focused on the development and quantification of resilience metrics, and some have proposed normative models for such systems. Common to their definitions is a concept of system-level coping capacity under multicomponent damage due to, for example: extreme meteorological conditions, geological hazards, and human-made events of an accidental or intentional nature. Damage to the system may also originate from less extraordinary events. In this context, resilience is generally conceived in terms of the system's ability to absorb damage thus continuing to serve the intended purpose, and recover within an acceptable time and cost (e.g., Holling 1973; Haimes 2009; The White House 2015; National Infrastructure Advisory Council 2015).

An underlying and typically unstated assumption in treating resilience is that the pre-event condition of all system components is uniform, fixed, known, and pristine. This means that resilience evaluations are, in effect, specific to the pre-event condition assumed at the moment of analysis as if the damage events were imminent. In reality, at a given point in time, the level of 'health' across components is uneven, with some offering a reduced inherent ability to endure damage. That is, infrastructure component integrity evolves over time. Two main governing and competing factors determine infrastructure integrity: (i) Progressive condition deterioration under usual service as a result of the combined effects of physical and environmental loading (i.e. "wear-and-tear" and aging), and (ii) Maintenance activities that aim for partial or complete condition renewal, or merely for slowing the natural deterioration rate (i.e., preventative). Based on a thorough review of the literature (Faturechi and Miller-Hooks 2015) which scanned over 200 articles, it appears that other than two works by Dehghani et al. (2013, 2014), no prior work on resilience or related performance measure computation has explicitly accounted for non-pristine integrity, timewise evolution, and subsequent unevenness in system component conditions. Dehghani et al. (2014) assessed expected network performance in terms of vehicle miles traveled and other devised vulnerability metrics over multiple randomly generated generic disruption scenarios involving link failures. Both papers discuss the need for condition-based vulnerability assessment as advocated herein, or more specifically, the need to incorporate each element's condition in replicating link failure probabilities. Their application in (Dehghani et al., 2014) 
on a hypothetical example demonstrates that a range over network performance results from assuming different link probability failure distributions (i.e. all link failures are either uniform, beta or normal). Herein, this general idea of a need for condition-based assessment is furthered.

This work proposes and demonstrates a condition-based resilience quantification methodology that incorporates component-condition evolution in a systems-based analysis. Resilience calculations, which may include resilience enhancing preparedness and response actions, are carried out under a set of possible damage-meteorological scenarios with known probability of occurrence. Each scenario consists of hazard type identification, meteorological state, number of affected segments (damage extent), and damage severity in terms of required repair duration and resources. The evaluation is performed a priori while accounting for optimal response actions that can be taken under each of the scenarios; preparedness actions that improve resilience are also optimized. To account for condition evolution, the health or integrity level of the individual system components immediately prior to hazard event impact (under all considered scenarios) is associated with a serviceability rating. This rating is projected to reflect both usual deterioration and policy-guided improvements due to maintenance. Impacts from generated damage-meteorological events are made to depend on the pre-impact serviceability ratings, exemplifying the added vulnerability of deteriorated components. The quantification scheme also captures the increased damage extent, extra repair costs, and longer repair times due to pre-event non-pristine conditions.

Hereafter, pavement condition and its expression through serviceability is first described. Then, an existing concept of resilience is restated and subsequently expanded to incorporate component serviceability rating. The formulation is applied next to an airport case study to demonstrate the value and effect of including natural deterioration and maintenance policies in resilience quantification. Lastly, gained insights and main conclusions of the study are listed and discussed. It is important to note that the applicability of the concepts and general methodology presented herein transcend this pavements application, generally applying to a system of components whose conditions differ, deteriorate over time and are influenced by maintenance and/or replacement actions. Specific models of deterioration/serviceability, maintenance or renewal planning, or system resilience as throughput or 
other will differ based on the use, but the framework is designed to be generally applicable. Moreover, it accounts for the system impacts of multiple link-based maintenance and resilience enhancements.

\section{Pavement Condition Evolution}

85

\section{The Serviceability Concept}

Without repair actions, pavements progressively deteriorate with time. The decline is directly associated with structural or physical damage involving distress modes, such as: rutting, cracking, longitudinal roughness, and raveling. It therefore represents a timewise diminishment in coping capacity against damage events of operational (e.g., overloading), natural (e.g. flooding), or other causes. The degradation pattern is case-specific as it depends upon the pavement design, as-built mechanical properties of the different layers, traffic intensity, and prevailing climatic conditions.

The concept of serviceability is often employed in the pavement arena for quantifying infrastructure condition. The idea was introduced and developed during the 1960's in conjunction with the AASHO road experiment (Carey and Irick 1960). In this experiment, different full-scale road sections were intensively trafficked for a period of two years by trucks of known weight, axle configurations, and travel speed. At the same time, the evolution of surface distress was closely monitored and recorded. A serviceability rating in the range of 5 (=pristine) to 0 (=worst possible), was adopted to quantify the condition of each road section from a user and structural perspective. Initially, the rating was based upon a subjective visual score given by a group of experts examining the ride surface (Present Serviceability Rating, PSR); it was later correlated with objectively measurable damage such as density of cracked or patched zones, longitudinal roughness, etc (Present Serviceability Index, PSI). In the AASHO road test, a power-law expression was found adequate for all pavement types to describe the evolution of serviceability as a function of traffic loadings:

$$
S=S_{i}-\left(S_{i}-S_{f}\right)\left(\frac{W}{\rho}\right)^{\beta},
$$

where $S$ denotes the current serviceability rating, $S_{i}$ is the initial serviceability rating prevailing immediately after construction, $S_{f}$ is the final or unacceptable serviceability rating, and $W$ is the 
cumulative number of equivalent vehicle passes applied to the section up to point for which $S$ is

108 calculated. In effect, if the traffic intensity is timewise uniform, $W$ may be seen as equivalent to age.

109 The parameters $\rho$ and $\beta$ are regression constants that embody the experimental setup, such as 110 pavement layering arrangement and mechanical properties, prevailing environmental conditions, and

111 loading characteristics of passing vehicles. By substituting $S=S_{f}$ it may be noticed that $\rho$ equals the 112 value of $W$ at failure. Graphically, the deterioration pattern (i.e., the shape of $S$ vs. $W$ curve) depends 113 upon the value of $\beta$; for $\beta=1$ the curve is an oblique line, for $\beta>1$ the curve is concave, while for $114 \beta<1$ it is convex. practice. At the same time it is deemed restrictive, incapable of correctly matching observed long-term pavement behavior, mainly because its curvature never reverses as needed (Fwa 1990). This shortcoming was later rectified by considering a slightly different equation capable of producing an Sshaped curve (Garcia-Diaz and Riggins 1984):

$$
S=S_{i}-\frac{S_{i}-S_{f}}{\exp \left((\tau / t)^{n}\right)}
$$

wherein $t$ is the time since construction or time elapsed since most recent repair (i.e. when serviceability is $\left.S_{i}\right), \tau$ and $n$ are parameters controlling the deterioration pattern, analogous to $\rho$ and $\beta$ in Equation 1. Note that $S=S_{f}$ is approached only at infinite time. By setting $S_{i}=100 \%$ (i.e., pristine) and $S_{f}=0$ (i.e., worst possible) the resulting serviceability curve can be viewed as a so-called survivor curve (Lytton 1987; Stampley et al. 1995).

Plots of Equation 2 over a 25 year period are included in Fig. 1. Three different $n$ values $(0.5$, 1.0, and 2.0) are considered in Fig. 1(a) for $\tau=7$ years. Three different $\tau$ values (5, 7 and 9 years) are considered in Fig. 1(b) for $n=1$. As can be seen, Equation 2 is able to describe a pavement that progressively deteriorates - while switching between three different degradation rates. Initially, for a certain time interval, the degradation rate is very small - allowing the pavement to practically remain in

131 pristine conditions. Next, the deterioration rate increases, causing a relatively quick drop in serviceability. Finally, the deterioration rate is arrested, slowing the drop in serviceability. In both charts the solid line indicates an assumed benchmark case, associated with $n=1$ and $\tau=7$ years. This 
benchmark case depicts a realistic situation for a pavement with an initial serviceability rating of $100 \%$ that deteriorates to $50 \%$ serviceability after 10 years and to a rating of about $25 \%$ after 25 years. Two pavement damage pictures are superposed over the charts, each associated with a different serviceability level. The association is approximate, merely provided to exemplify the physical meaning of the curves. The purpose here is to intuitively reinforce the link between lower serviceability and infrastructure vulnerability to a hazard event.

Also included in Fig. 1 are qualitative ratings of infrastructure condition, ranging from "Good" to "Failed". The descriptive scale is identical to that employed in the Pavement Condition Index (PCI) method (ASTM D6433 or ASTM D5340), a scheme often used by pavement managers (Shahin 2005).

143 For the purpose of the current work a PCI index may be used in place of a serviceability rating, i.e., they 144 are interchangeable.

\section{Maintenance}

146 The curves in Fig. 1 essentially represent pavement condition under a no-maintenance situation. When

147 maintenance is applied at some point in time, it changes the shape of the deterioration curve. First, 148 rehabilitation activities appear as a sudden jump in the curve, i.e., an abrupt increase due to improved 149 serviceability. Further, maintenance work alters the subsequent shape of the deterioration curve. 150 Realistically, pavements cannot be preserved at their original as-built serviceability levels throughout 151 the life of the system. Hence, some decline in performance is allowed in the different network 152 components before taking repair actions. Policies for maintaining deteriorating systems have been 153 studied extensively (not necessarily for the pavement discipline), with numerous proposed model types 154 (Wang 2002), e.g., age-based, periodic/sequential, failure/performance/condition-based, cost limited, 155 repair-duration limited, opportunistic, etc. et al. 2011; Air Force Civil Engineer Center 2014). Typically, a set of intervention rules is pre-stated,

158 triggering a specific maintenance effort on the basis of the component condition level. An intervention rule associated with a high serviceability rating usually requires more frequent repairs but involves only

160 preventive or minor rehabilitation. Triggering intervention at low serviceability levels typically implies that major and costly rehabilitation efforts are necessary. Research on this connection, e.g. Camahan et 
al. (1987), Madanat and Ben-Akiva (1994), has generally focused on maximizing cost effectiveness by

163 optimizing decisions, such as: (i) how frequently maintenance should be applied; (ii) to what condition

164 a pavement should be allowed to deteriorate before action is taken; and (iii) what best maintenance alternative to take for a given situation. Contributions in this field have also attempted to integrate condition forecasting into the optimization.

Fig. 2 depicts condition evolution for a pavement under a simplistic threshold-based maintenance policy. The abscissa represents time and the ordinate represents serviceability rating. Starting from an arbitrary "current" rating, the condition is seen to degrade with elapsed time until a predefined threshold level is reached ( $S=60 \%$ in the case shown). This threshold designates a minimal acceptable serviceability rating for the infrastructure being considered. Repair intervention is therefore triggered, raising the rating to $S=100 \%$. Then after, the pavement condition continues its decline, triggering a new repair intervention once the threshold is encountered again. This situation is repetitive/cyclic. The depiction in Fig. 2 is deemed simplistic, because it presumes that the deterioration curve has identical shape before and after repair, and because the pavement receives treatment at the exact designated timing - assuming all necessary resources are available for the repair. Without loss of generality, these simplifications are adopted for the current work.

\section{Resilience Definition}

180 The literature is replete with qualitative discussions and quantitative methods for measuring system

181 resilience, as well as other related system performance metrics, including: risk, vulnerability, reliability, robustness, flexibility, survivability, etc. See Faturechi and Miller-Hooks (2015) for a synthesis of articles proposing such measures in the context of transportation systems alone. The concept of resilience as adopted and computed herein considers two main features: (i) the system's innate ability, based on the physical properties and topology/connectivity of its components, to cope with a disruption event that causes physical damage and (ii) the system's ability to adapt through quick, cost-effective actions that can preserve or restore post-event performance/functionality. Both features are depicted in

188 Fig. 3, which schematically illustrates system performance vs. time before and after a disruption event; notice the time axis changes scale between pre- and post-event, from months (pre-disruption) to hours 
(upon disruption). As can be seen, the system's post-event performance level after a time period with length $T^{\max }$ is composed of two parts: (i) coping capacity - defined as performance level prevailing immediately after disruption; and (ii) adaptive capacity - defined as the improvement in performance level restored during $T^{\max }$ for a given set of repair resources. Ultimately, resilience is defined with respect to a baseline. It is taken as the ratio of post-response system performance level at event time $t$ to pre-event system performance level for link serviceability levels at base time zero.

Fig. 3(a) illustrates the commonplace assumption of a system that is initially (and at all times) in pristine condition (e.g., Bruneau et al. 2003). On the other hand, Fig. 3(b) depicts a system with fluctuating performance. This latter case is the non-traditional viewpoint offered herein; it originates from evolving component conditions and varying component age. As can be seen, pre-event performance level for the system is imperfect, leading to poorer coping and adaptive capacities than seen in Fig. 3(a).

A mathematical modeling approach proposed in Miller-Hooks et al. (2012) is used in this paper, wherein resilience is quantified through solution of a nonlinear, two-stage, stochastic program. The stochastic program seeks to maximize the expectation of an indicator representing the resilience of the network (throughput in this case) over possible disruption scenarios. That is, resilience actions are incorporated in the resilience computation, thus accounting for not only the innate coping capacity of the system, but also post-event adaptability in the disruption event's immediate aftermath. Optimal mitigation and preparedness actions are determined in the first-stage prior to event realization, and scenario-dependent, optimal, remedial actions are chosen in the second-stage in the form of recourse. Recourse decisions are taken with full knowledge of how the event is realized. Availability, cost and implementation time of recourse options may also depend on the choice of preparedness actions. An integer L-shaped decomposition method is applied to provide exact solution for the problem. This method decouples first- and second-stage decisions, eliminating bilinear terms that are the root of the nonlinearity.

As a means of introducing evolving component conditions into the resilience model, failure probabilities are associated with serviceability, the latter being governed by: age, normal deterioration curves, and maintenance policies. Specifically, the probability an adverse event will lead to component 
failure depends on the component's serviceability level at the event time, and the ability of the system

219 to provide services depends on its functioning components. Moreover, the cost and time for

220 implementing repair or other restorative options post-event are also functions of pre-event serviceability

221 rating. If a component is deteriorated, the effort required to return it to pristine condition will be greater

222 than if that component were not deteriorated. This is in part because the effectiveness of the restorative

223 options will be diminished once the component has deteriorated to certain levels. This integration of 224 component condition in resilience computation is described and demonstrated through a case study 225 given next.

\section{Demonstration on Case Study}

\section{Introduction and Design}

A specific case study is employed hereafter to demonstrate and assess the effects and value of incorporating evolving component conditions in resilience quantification. Use is made of a pavement system representing Laguardia Airport's (LGA's) taxiway and runway network. This choice builds on previous work (Faturechi et al. 2014), which was motivated by the fact that air transportation is one of fastest growing transportation modes worldwide. It is also driven by the particular sensitivity of airport operations to pavement condition. LGA contains two intersecting runways and supporting taxiways - as depicted in Fig. 4. These components and their interconnectivity are represented by a 68-node, 104-link network.

Resilience of the LGA pavement network was computed in Faturechi et al. (2014) with an implicit assumption of pristine component condition (pre-event). Fig. 5 synopsizes the employed modeling approach, and the reader is encouraged to consult Faturechi et al. (2014) for full details. In general terms, the model involves identification of a set of potential directed paths through the airport network for maneuvers between the gates and takeoffs or landings. Despite that each runway can be

242 used in two directions, within a given period of time, runway operations are unidirectional for safety 243 reasons. Thus, the model forces a choice of direction, a so-called runway configuration, within a given 244 time period. If an arc is damaged, its capacity to support the movement of an aircraft is zero; hence, only paths whose constituent arcs are undamaged or repaired can support flow. 

computed as the ratio $\alpha_{B, T^{\max }}$ of the expected number of landings and takeoffs to a comparable predisruption flow rate given by demand $D^{w, s}$ for each maneuver $w$ and aircraft type $s$, over all disaster scenarios $\xi$. As defined in Figure $5, f_{p}^{g, w, s}(\xi)$ is the flow rate of maneuver $w$ for aircraft type $s$ in path $p$ under runway configuration $g$ and scenario $\xi$.

$$
\alpha_{B, T^{\max }}=\frac{E_{\tilde{\xi}}\left\lfloor\sum_{w, g, s} \sum_{p \in P^{g, w, s}} f_{p}^{g, w, s}(\xi)\right\rfloor}{\sum_{w, s} D^{w, s}},
$$

252

The expectation is taken over a set of predicted disaster-meteorological event scenarios that may arise due to any one of a number of hazard event types, whether natural, accidental or malicious, with anticipated occurrence probabilities. First-stage decisions mitigate disaster impact and can support postevent repair opportunities (e.g. availability of materials, repair equipment, trained crews, and contracts with external resources). These decisions are be taken a priori with the knowledge that second-stage recourse (repair) decisions will be taken optimally a posteriori given the available resources, and knowledge of how the disaster-meteorological event unfolds.

An overview of the computational framework, specified for the case study, is given in Fig. 6. Similar to Faturechi et al. (2014), the scheme consists of three main modules: (i) scenario generation, (ii) mathematical modeling; and (iii) model solution. As part of the scenario generation, runway and taxiway link failure probabilities were made functions of serviceability as described in Equation 2. To capture serviceability levels as a function of component's age and maintenance plan, serviceability ratings $S^{a}(t)$ were explicitly defined as a function of time $t$ for each runway or taxiway link $a$ :

$$
S^{a}(t)=S_{i}^{a}-\frac{S_{i}^{a}-S_{f}^{a}}{\exp \left(\left(\frac{\tau}{t-t_{m}^{a}}\right)^{n}\right)},
$$

where $t_{m}^{a}$ is the time of last maintenance before time $t$ which has brought the serviceability of link $a$ back to $S_{i}^{a}$ at that time ( $t>t_{m}^{a}$ at all times). $S_{i}^{a}$ is the initial serviceability condition typically taken as pristine, and $S_{f}^{a}$ is the final (worst possible or ultimate) serviceability rating approached if no repair is applied typically taken as zero. 
Each scenario is defined in terms of damage severity and type (climate/geological, operational, natural deterioration, and terrorism), along with current meteorological conditions in terms of temperature, precipitation and visibility conditions. Meteorological conditions are described in terms of temperature, visibility, wind velocity and precipitation, which might affect potential damage causes and types. The causes also affect damage location and distribution of damage in multiple locations over the pavement network. The likelihood of an event falling within any of these causal categories depends on the geographical characteristics of the airport. A host of damage-weather scenarios are possible. To capture correlations between damage characteristics and meteorological conditions, conditional probabilities are employed in generating scenarios. The probability of each scenario is assumed to be known a priori. Specifically, let $p_{a}\left(\xi_{0}\right), p\left(\xi_{0} \mid d\right), p(d \mid m)$ and $p(m)$, be the probability of scenario $\xi_{0}$, probability of scenario given disruption type $d$, probability of disaster type given meteorological condition $m$, and probability of meteorological condition $m$ for the given geographical location, respectively. Then,

$$
p_{a}\left(\xi_{0}\right)=p(s \mid d) \cdot p(d \mid m) \cdot p(m)
$$

The failure probability $p_{a}\left(\xi_{t}\right)$ of link $a$, given disruption-meteorological event $\xi_{t}$ arising at time $t$, is related to $S^{a}(t)$ as follows:

$$
p_{a}\left(\xi_{t}\right)=\min \left\{1,\left(1+c\left(1-S^{a}(t)\right)\right) p_{a}\left(\xi_{0}\right)\right\}
$$

where $p_{a}\left(\xi_{0}\right)$ is the failure probability of link $a$ at $100 \%$ serviceability (i.e. under pristine conditions) for given event type. As can be seen, the sought failure probability is governed by a positive proportionality constant $c$. This is a newly introduced parameter that links serviceability ratings to the formulation. Higher values of $c$ infer greater influence of component condition on failure probabilities. Note that if $c$ is set large enough, it is possible that a probability greater than one would be generated; a ceiling of probability-one is therefore assumed. If $S^{a}(t)=100 \%$ at all times, then the influence of $c$ is annulled, yielding the familiar assumption in the resilience literature: $p_{a}\left(\xi_{t}\right)=p_{a}\left(\xi_{0}\right)$. Similarly, 294 the damage severity of link $a$ is captured through repair action implementation cost and time, which are also formulated as a function of the link's serviceability level:

$$
b_{a}\left(\xi_{t}\right)=\left(1+c\left(1-S^{a}(t)\right)\right) b_{a}\left(\xi_{0}\right)
$$




$$
q_{a}\left(\xi_{t}\right)=\left(1+c\left(1-S^{a}(t)\right)\right) q_{a}\left(\xi_{0}\right)
$$

in which $b_{a}\left(\xi_{0}\right)$ and $q_{a}\left(\xi_{0}\right)$ are implementation cost and time of repair actions in link $a$ with a serviceability rating of $100 \%$. Their values depend on the event type. While the $c$ parameter was taken as identical across Equations 6 and 7, in a more general formulation this presumption may be relaxed.

Given possible weather conditions and probability of their occurrences specific to LGA, disruption-meteorological events, resulting damage types (e.g. cracking, disintegration, distortion, loss of skid resistance), potential damage extent in terms of maximum number of affected segments, and repair actions required in each case were generated. Each disruption meteorological event gives a set of link failure probabilities which are used to randomly generate operational (one) or failed (zero) link states to create each disruption-meteorological scenario. An overview of the scenario generation process is given in Fig. 6. Conditional probabilities capture correlations between damage characteristics and meteorological conditions. Thus, the result of scenario generation is the set of disruption-meteorological events with one/zero values for link functionality and characteristics associated to that disruption event such as the required repairs, available repairs, etc. Ultimately, resilience was assessed at 6-month intervals over a 15 -year time horizon during which network component conditions continually evolved.

\section{Case Study Specifics}

Presented in what follows are modeling details involved in resilience quantification of the LGA case study. First, a budget $B$ for emergency preparedness and response of $\$ 25,000$ was assumed. Also, $T^{\max }$ was set to 8 hours, and $c$ in Equations 6 and 7 was taken to equal 1.5 (in lieu of relevant information from other sources this choice was based on preliminary run results). Resilience is measure of a system's innate coping capacity and ability to adapt when confronted with a challenge. Thus, resilience is conceptualized here to include adaptive actions that can be taken quickly and relatively cheaply. Higher monetary and time budgets can be used; however, a system that would require significant resources for continued operations might not be considered resilient. Benchmark deterioration curve parameter set was assumed to hold for both taxiways and runways; with reference to Equation 4 these are $n=1$ and $\tau=7$ years. Two separate threshold-based maintenance plans (MPs) were considered. In MP1 rehabilitation actions are taken whenever runway serviceability reaches $80 \%$ and taxiway serviceability reaches $60 \%$. This is consistent with a repair interval of about 4.0 and 7.5 
years, respectively. In MP2 the rehabilitation thresholds were $60 \%$ for runways and $40 \%$ for taxiways. Respectively, these imply repair intervals of about 7.5 and 13.5 years.

With both MPs, runways are maintained at higher average levels than are taxiways. MP1 imposes more stringent rehabilitation demands as compared to MP2, and represents an airport pavement network that is, on average, in better condition. Moreover, the age of each runway at the beginning of the resilience analysis period was randomly set given $\sim \mathrm{U}[0,4.0]$ and $\sim \mathrm{U}[0,7.5]$ years for MP1 and MP2, respectively. Similarly, the starting age of the taxiways was randomly set given $\sim \mathrm{U}[0,7.5]$ and $\sim \mathrm{U}[0,13.5]$ years for MP1 and MP2, respectively. This procedure generated a realistic situation where the serviceability across the network is non-uniform.

MP1 and MP2 parameters are summarized in Table 1 which lists the initial ages of the different network components, as well as their associated serviceability rating and maintenance threshold. As may be seen, taxiways were grouped based on their orientation relative to the runways: parallel and perpendicular. Such distinction has some operational implication that is captured (internally) by the model. Condition evolution of taxiways and runways according to MP1 is plotted in Fig. 7(a). Similar information for MP2 is included in Fig. 7(c). Each chart includes four lines, representing changes in infrastructure serviceability over a 15 year period. Starting levels are dissimilar per Table 1 values. As can be seen, full rehabilitation to pristine conditions is presumed after a threshold is encountered, generating a repetitive pattern. Because starting serviceability levels are different, and because the rehabilitation threshold for taxiways and runways are different, the condition of the system at any point in time is spatially nonuniform.

\section{Results and Analysis}

The resilience indicators for the case study, calculated through Equation 3, are presented in Fig. 7. Charts 7(b) and 7(d) display resilience calculation outcomes associated with MP1 (Fig. 7(a)) and MP2 (Fig. 7(c)), respectively. Each chart contains 31 values covering an analysis period of 15 years at 6-month intervals. The resilience values fluctuate due to differences in component conditions between the different evaluation times, and also because of the statistical nature of generating scenarios. Specifically, each point in the figure is computed from an average performance value over 360 randomly generated disruption-meteorological scenarios. Model runs might be repeated over additional sets of randomly 


\section{Conclusions and Future Work}

373 This paper is concerned with quantifying the resilience of an airport pavement network while allowing

374 for evolving component conditions. Application to a case study demonstrated that resilience is impacted 375 by the initial condition of the infrastructure links, by their natural deterioration trends, and by prevailing maintenance policies and actions. The impact found was not negligible, indicating the need and value

generated scenarios to produce a range of resilience estimates or a single expectation over a larger set of possibilities.

Two horizontal lines are superposed on each resilience chart, forming bands that encapsulate all run results. These lines represent a single upper bound (UB) evaluation and a single lower bound (LB) evaluation of the system resilience plus (or minus) two standard deviations that were calculated based on the spread in each case. The UB case denotes system resilience level with all components in pristine condition (pre-event). It is therefore unaffected by MP specifics. The LB case denotes a system resilience level with all components at their worst allowable condition simultaneously - according to the governing MP threshold. This LB value will differ between MPs and in the case shown is slightly higher for the more stringent MP1. Note that while pristine conditions are presumed in the computation of the resilience upper bound, and worst acceptable serviceability for the lower bound, the resilience bound values are computed over 360 randomly generated disruption-meteorological scenarios. Thus, they may vary as a function of the scenario generation output. The difference between UB and LB is about 17\% for MP1 and about 20\% for MP2. This difference directly depends on the value chosen for $c$ in Equations 6 and 7 and the set of generated scenarios.

Overall, Fig. 7 reveals that the network resilience changes over time between the upper and lower bounds with values that depend, among other factors, on link conditions, link natural deterioration pattern, and prevailing MPs. for such an approach. The method employed is flexible and can be further refined or compounded by, for example: (i) assigning different maintenance thresholds to different components or incorporating other repair policies; (ii) optimizing maintenance actions rather than assuming a given schedule or 
protocol; or (iii) using any one of a number of serviceability models, including stochastic methods for

381 predicting future condition.

Note that for other pavement networks, such as a roadway network, the adopted resilience metric would be modified. In the case of the roadway network, a measure based on vehicular throughput or travel time/delay could be employed. In the latter case, a bi-level programming formulation might be adopted where the lower level would provide link travel time estimates given post-event roadway conditions and chosen resilience actions. Refer to Faturechi and Miller-Hooks (2015) for roadway resilience estimation in which pristine conditions are implicitly assumed; such estimates account for user response to system changes.

Even though a specific case and type of application were considered, the findings here are of general nature; they imply that earlier resilience works report UB values (refer to Fig. 7). In other words, best-case resilience estimates are typically provided. For the current formulation this is equivalent to annulling $c$ in Equations 6 and 7. Moreover, in light of evolving component conditions, the definition of resilience may also require reexamination. Resilience is typically quantified relative to a pre-event baseline signifying pristine system performance. Because component conditions are allowed to evolve, pristine performance is not realistically achievable, while at the same time pre-event performance fluctuates (see Fig. 3).

Commonly, optimization of MPs is based on life cycle cost analyses. A continuation of this work may include MPs that are associated with resilience quantification, i.e., investigating MPs in terms of effects on resilience. One option in this connection is making the MP a decision variable, with its own budget, and integrating in the decision process for preparedness (current model did not include maintenance cost and resources). Timing and location of repair decisions was not considered in the employed MP, but the approach here allows testing such strategies (e.g., Medury and Madanat 2013).

403 So doing can lead to new implications for maintenance budget allocation/prioritization. Also of interest 404 is performing an in-depth parametric/sensitivity analysis of each resilience calculation. This means investigating the solution details for resilience by event categories, differences in division of budget between preparedness and response, or any other changes in decision variables. These aspects will serve as topics for future work. 


\section{Acknowledgements}

This work was funded by the National Science Foundation. This support is gratefully acknowledged, but implies no endorsement of the findings.

\section{References}

Air Force Civil Engineer Center. (2014). "Engineering Technical Letter (ETL) 14-3: Preventive Maintenance Plan (PMP) for Airfield Pavements.” Department of the Air Force, Tyndall Air Force Base, Florida.

Bruneau, M., Chang, S. E., Eguchi, R. T., Lee, G. C., O'Rourke, T. D., Reinhorn, A. M., Shinozuka, M., Tierney, K., Wallace, W. A., and von Winterfeldt, D. (2003). "A framework to quantitatively assess and enhance the seismic resilience of communities." Earthquake Spectra, 19(4), 733-752.

Camahan, J., Davis, W., Shahin, M., Keane, P., and Wu, M. (1987). "Optimal maintenance decisions for pavement management.” Journal of Transportation Engineering, 113(5), 554-572.

Carey, W. N., Jr., and Irick, P. E. (1960). “The pavement serviceability-performance concept.” Highway Research Board Bulletin, 250, 40-58.

Dehghani, M. S., Flintsch, G. W., and McNeil, S. (2013). "Roadway network as a degrading system: vulnerability and system level performance." Transportation Letters, 5(3), 105-114.

Dehghani, M. S., Flintsch, G. W., and McNeil, S. (2014). "Impact of road conditions and disruption uncertainties on network vulnerability.” Journal of Infrastructure Systems, 20(3), 04014015.

Faturechi, R., Levenberg, E., and Miller-Hooks, E. (2014). "Evaluating and optimizing resilience of airport pavement networks." Journal of Computers and Operations Research, 43, 335-348.

Faturechi, R., and Miller-Hooks, E. (2015). "Measuring the performance of transportation infrastructure systems in disasters: a comprehensive review.” Journal of Infrastructure Systems, 21(1), 1-15.

Federal Highway Administration Pavement Distress Identification Definition Manual, wistrans.org/mrutc/files/Distress-ID-Manual.pdf (accessed on June 17, 2015).

Fwa, T. F. (1990). "Shape characteristics of pavement performance curves." Journal of Transportation Engineering, 116(5), 692-697.

Garcia-Diaz, A., and Riggins, M. (1984). "Serviceability and distress methodology for predicting pavement performance.” Transportation Research Record, 997, 56-61.

Haimes, Y. Y. (2009). “On the definition of resilience in systems.” Risk Analysis. 29(4), 498-501.

Hajek, J., Hall, J. W., and Hein, D. K. (2011). “Common airport pavement maintenance practices.” A Synthesis of Airport Practice, ACRP SYNTHESIS 22, Federal Aviation Administration, Transportation Research Board, Washington, D.C.

Holling, C. S. (1973). "Resilience and stability of ecological systems." Annual Review of Ecology and Systematics, 4, 1-23.

Lytton, R. L. (1987). "Concepts of pavement performance prediction and modeling." Proceedings of the 2nd North American Conference on Managing Pavements, Ontario, Ministry of Transportation and U.S. Federal Highway Administration, Toronto, Ontario, Canada, 2.3-2.20.

Madanat, S., and Ben-Akiva, M. (1994). "Optimal inspection and repair policies for infrastructure facilities." Journal of Transportation science, 28(1), 55-62.

Medury, A., and Madanat, S. (2013). Incorporating network considerations into pavement management systems: a case for approximate dynamic programming." Transportation Research Part C, 33, 134-150. 
450 Miller-Hooks, E., Zhang, X., and Faturechi, R. (2012). "Measuring and maximizing resilience of freight 451 transportation networks." Computers and Operations Research, 39(7), 1633-1643.

452 National Infrastructure Advisory Council, Critical Infrastructure Resilience Final Report and 453 Recommendations. http://www.dhs.gov/xlibrary/assets/niac/niac_critical_infrastructure_resilience.pdf 454 (accessed July 27, 2015).

455 Shahin, M. Y. (2005). "Pavement management for airports, roads, and parking lots." Springer, 572 p.

456 Stampley, B., Miller, B., Smith, R., and Scullion, T. (1995). "Pavement management information system 457 concepts, equations and analysis models." Texas Transportation Institute, Research Report TX 96/1989$458 \quad 1$.

459 The White House, Office of the Secretary, Presidential Policy Directive -- Critical Infrastructure Security 460 and Resilience, Presidential Policy Directive/PPD-21, 2013. https://www.whitehouse.gov/the-press461 office/2013/02/12/presidential-policy-directive-critical-infrastructure-security-and-resil (accessed 462 July 27, 2015)

463 Wang, H. (2002). "A survey of maintenance policies of deteriorating systems." European Journal of 464 Operational Research, 139(3), 469-489. 
469 Table 1. Details of Maintenance Plans

\begin{tabular}{|c|c|c|c|c|c|c|}
\hline \multirow[b]{2}{*}{$\begin{array}{l}\text { Infrastructure } \\
\text { Component }\end{array}$} & \multicolumn{3}{|c|}{ Maintenance Plan 1 (MP1) } & \multicolumn{3}{|c|}{ Maintenance Plan 2 (MP2) } \\
\hline & $\begin{array}{c}\text { Starting } \\
\text { age } \\
\text { (years) }\end{array}$ & $\begin{array}{c}\text { Starting } \\
\text { Serviceability } \\
\text { rating } S_{a}(0)\end{array}$ & $\begin{array}{c}\text { Predefined } \\
\text { Repair } \\
\text { Threshold }\end{array}$ & $\begin{array}{l}\text { Starting } \\
\text { age } \\
\text { (years) }\end{array}$ & $\begin{array}{c}\text { Starting } \\
\text { Serviceability } \\
\text { rating } S_{a}(0)\end{array}$ & $\begin{array}{c}\text { Predefined } \\
\text { Repair } \\
\text { Threshold }\end{array}$ \\
\hline Runway 1 & 3.1 & $90 \%$ & \multirow{2}{*}{$80 \%$} & 3.8 & $84 \%$ & \multirow{2}{*}{$60 \%$} \\
\hline Runway 2 & 1.7 & $98 \%$ & & 1.1 & $100 \%$ & \\
\hline Taxiway-perpendicular & 4.5 & $79 \%$ & \multirow{2}{*}{$60 \%$} & 10.5 & $49 \%$ & \multirow{2}{*}{$40 \%$} \\
\hline Taxiway-parallel & 6.2 & $68 \%$ & & 3.2 & $89 \%$ & \\
\hline
\end{tabular}

470

471 


\section{List of Figure Captions}

Fig. 1. Serviceability curves (Eq. (2)) showing the influence of: (a) $n$ parameter, and (b) $\tau$ parameter. Superposed damage pictures illustrate the physical meaning of condition rating; image source: Federal Highway Administration Pavement Distress Identification Definition Manual (2015)

Fig. 2. Illustration of a threshold-based maintenance policy

Fig. 3. Approaches to infrastructure resilience: (a) pre-event system performance is timewise constant with all components in pristine condition; and (b) pre-event system performance fluctuates due to non-uniform component conditions

Fig. 4. LGA runway and taxiway network layout

Fig. 5. Overview of stochastic program for airport pavement network resilience computation employed in Faturechi et al. (2014)

Fig. 6. Diagram of case study resilience quantification

Fig. 7. Case study results: (a) evolution of serviceability according to MP1, (b) consequent system resilience under MP1, (c) evolution of serviceability according to MP2, and (d) consequent system resilience under MP2 

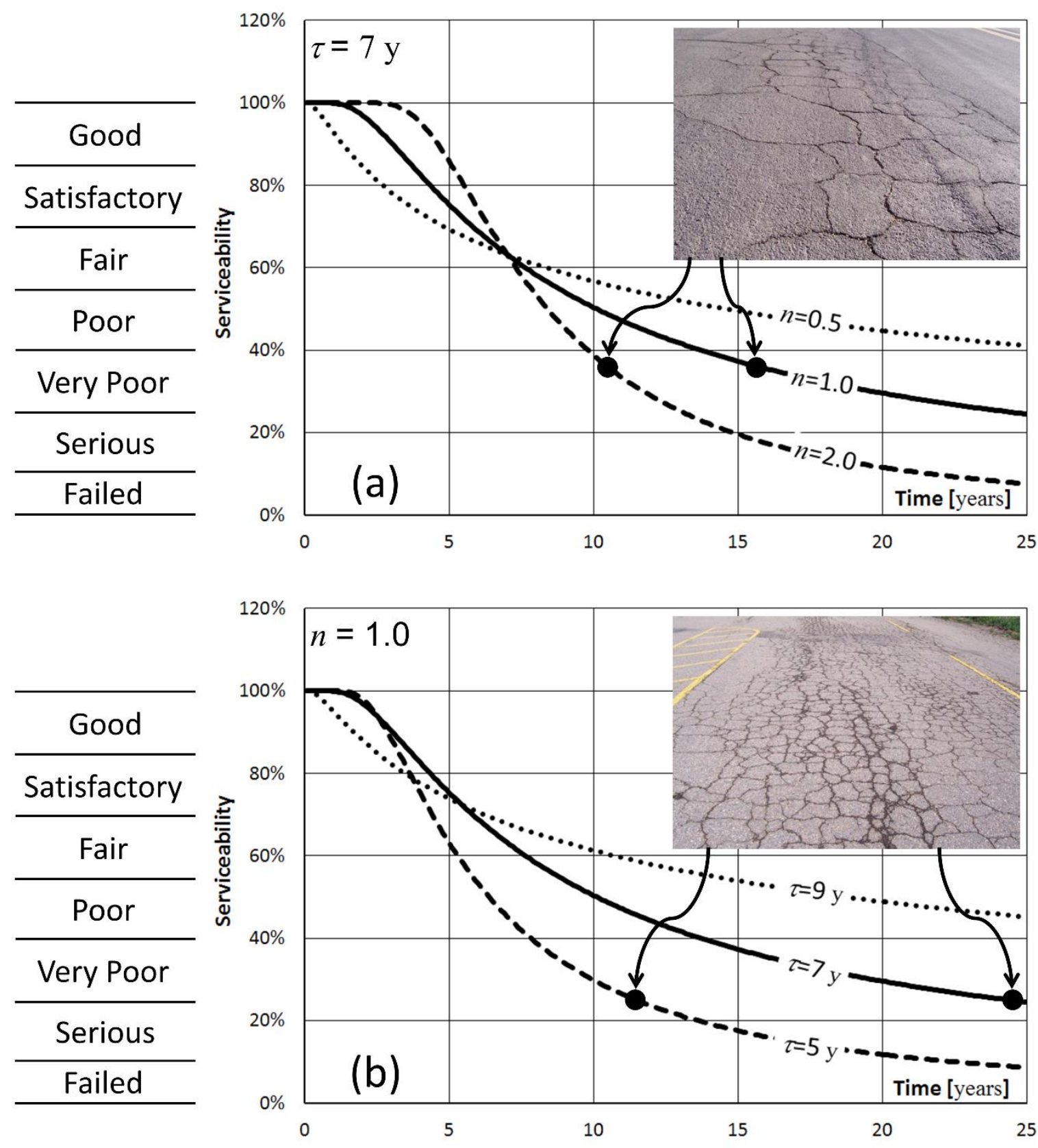

Fig. 1. Serviceability curves (Eq. (2)) showing the influence of: (a) $n$ parameter, and (b) $\tau$ parameter. Superposed damage pictures illustrate the physical meaning of condition rating; image source: 


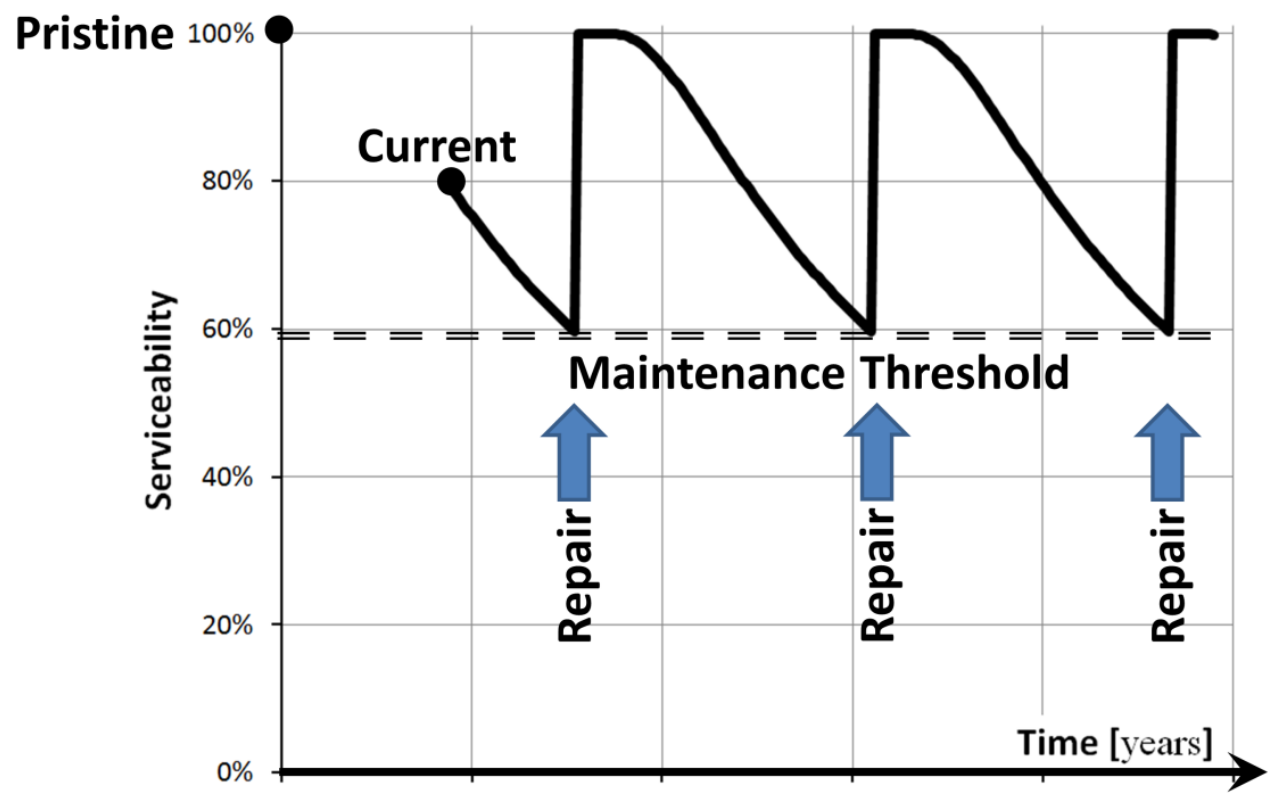

498 Fig. 2. Illustration of a threshold-based maintenance policy 

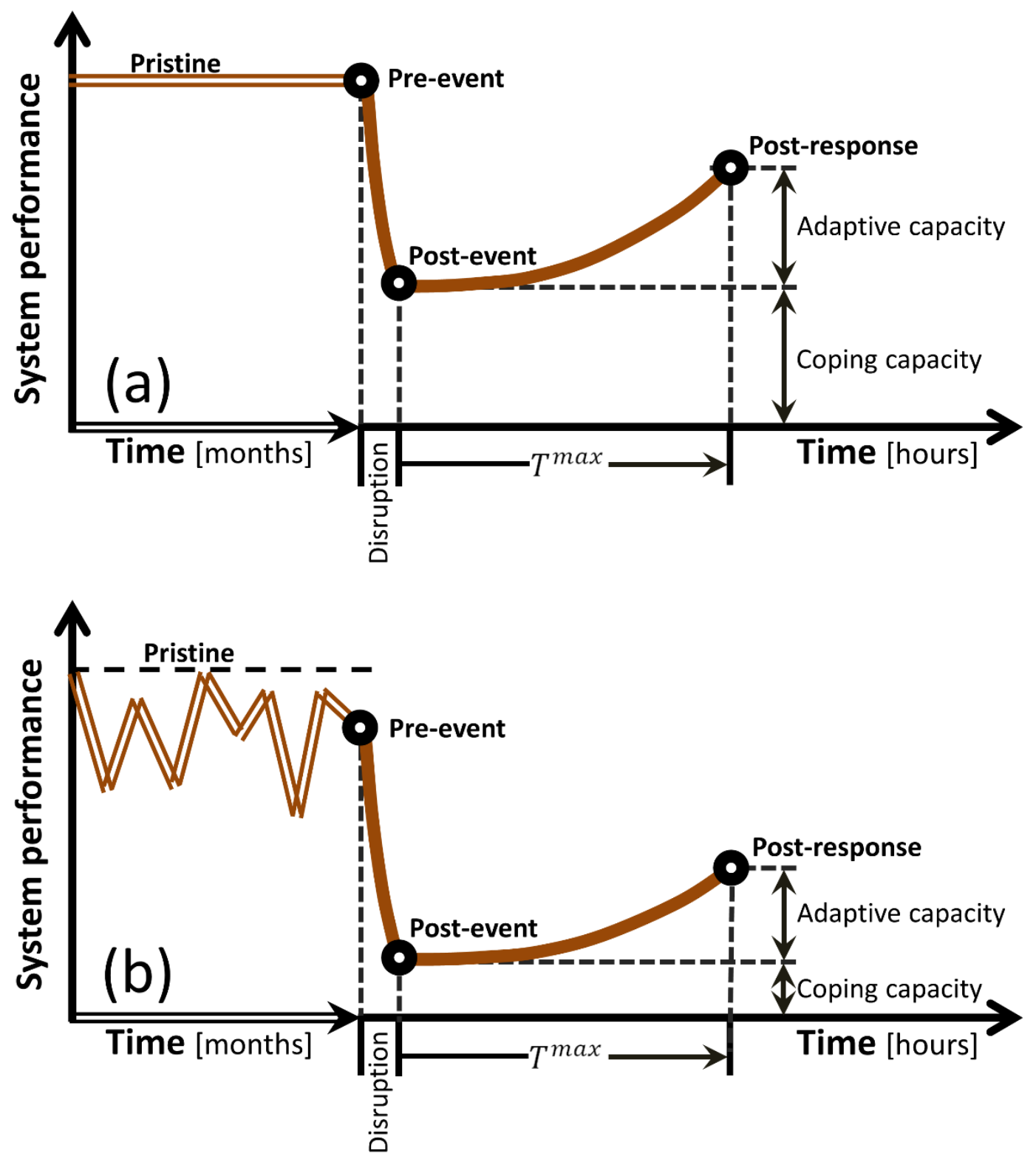

Fig. 3. Approaches to infrastructure resilience: (a) pre-event system performance is timewise constant 503 with all components in pristine condition; and (b) pre-event system performance fluctuates due to non-uniform component conditions 


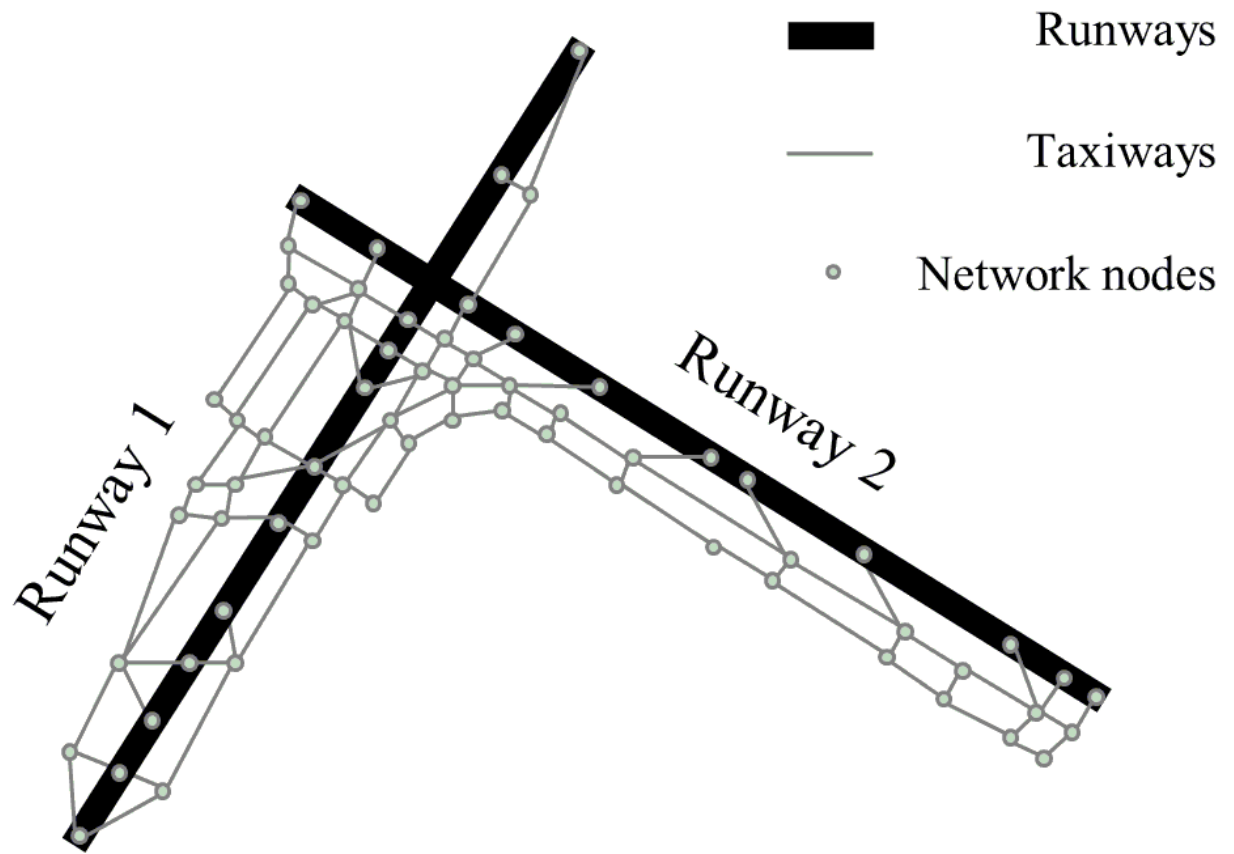

Fig. 4. LGA runway and taxiway network layout 


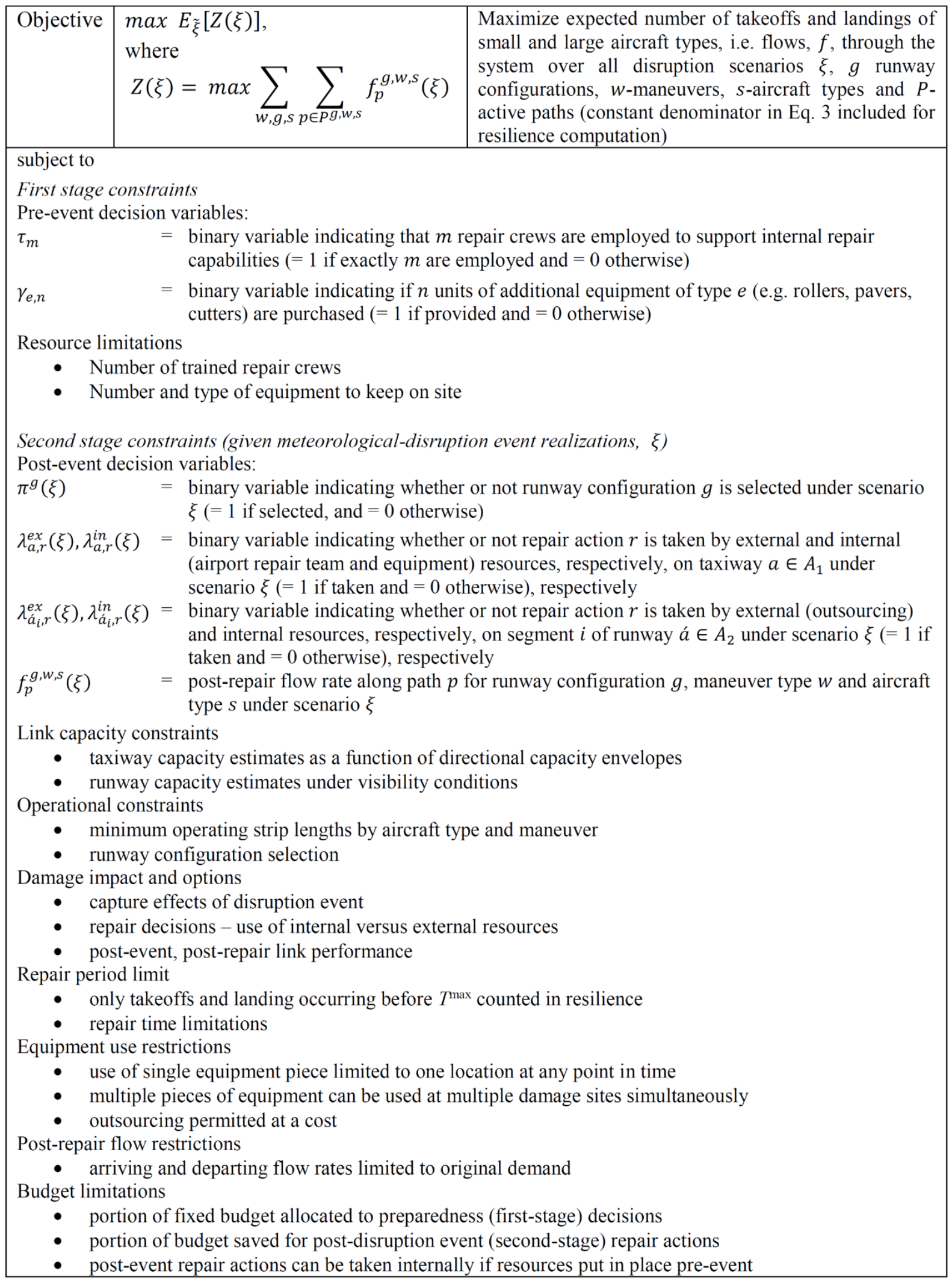

Fig. 5. Overview of stochastic program for airport pavement network resilience computation employed in Faturechi et al. (2014) 


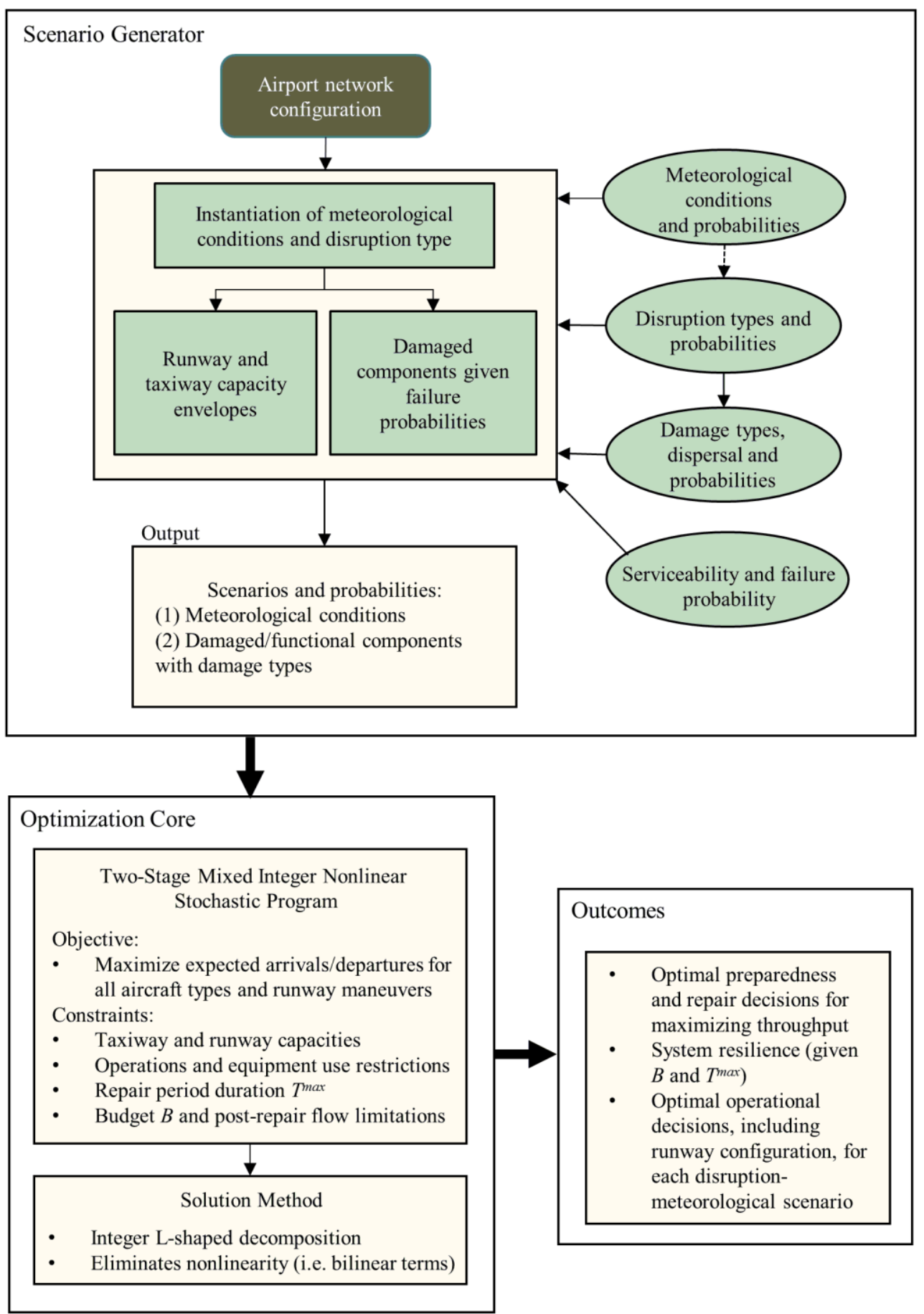

515 Fig. 6. Diagram of case study resilience quantification 

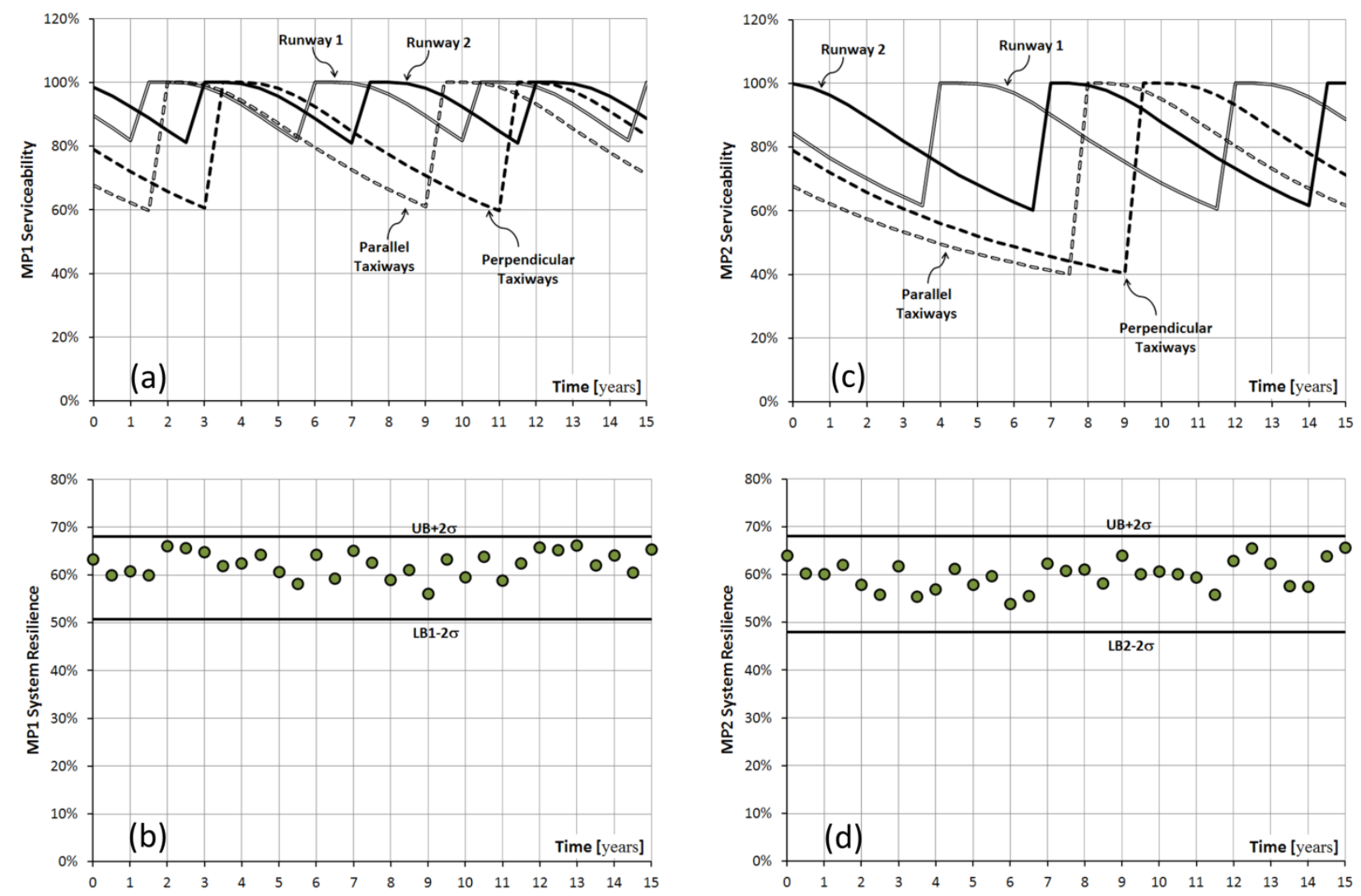

Fig. 7. Case study results: (a) evolution of serviceability according to MP1, (b) consequent system resilience under MP1, (c) evolution of serviceability according to MP2, and (d) consequent system resilience under MP2 